ment, the random uncertainty for the average value is reduced to $1.1 \times 10^{-8}$.

\section{CONCLUSION}

A new method of quantum Hall effect measurement has been developed utilizing a Josephson potentiometer and a SQUID galvanometer. It is demonstrated that measurement with an uncertainty of 2.5 parts in $10^{8}$ is possible when this method is used for the measurement of the ratio of the $i=4$ quantized Hall resistance to a $6.45-\mathrm{k} \Omega$ reference resistor. The ratio measurement of the quantized Hall resistance to a $10-\mathrm{k} \Omega$ reference resistor has been successively performed by using this method. This method will be useful for investigating the universality of the quantum Hall effect to high precision.

\section{ACKNOWLEDGMENT}

The authors wish to thank to Dr. A. Yagi, Sony Corporation, for providing the Si-MOSFET samples, and Prof. S. Kawaji and Dr. J. Wakabayashi, Gakushuin University for useful discussions. They also wish to thank Dr. T. Dai and his colleagues at Takeda Riken Industry Co. Ltd. for providing reference resistors.

\section{REFERENCES}

[1] C. Yamanouchi, K. Yoshihiro, J. Kinoshita, K. Inagaki, J. Moriyama, S. Baba, S. Kawaji, K. Murakami, T. Igarashi, T. Endo, M.
Koyanagi, and A. Nakamura, "Hall effect in silicon MOS inversion layers for $\mathrm{h} / \mathrm{e}^{2}$ determination," in Proc. Precision Measurement and Fundamental Constants II, NBS Spec. Publ. 617, pp. 529-534, 1984.

[2] J. Kinoshita, K. Inagaki, C. Yamanouchi, K. Yoshihiro, T. Endo, Y. Murayama, M. Koyanagi, J. Moriyama, J. Wakabayashi, and S. Kawaji, "Precision measurement of quantum Hall effect in silicon Mos n-inversion layers," in Proc. Int. Symp. Foundations of Quantum Mechanics, Tokyo, Japan, pp. 339-345, 1983.

[3] E. Braun, F. Staben, and K. von Klitzing, "Experimental determination of $h / \mathrm{e}^{2}$ by the quantized Hall resistance in MOSFETs," PTB-Mitt., $905 / 80$, pp. 350-351, 1980; A correction is given in E. Braun, P. Gutmann, G. Hein, F. Melchert, P. Warnecke, S. Xue, and K. von Klitzing, "Cryogenic method for the determination of the fine structure constant by the quantized Hall resistance, in Proc. Precision Measurement and Fundamental Constants II, NBS Spec. Publ. 617, pp. 535-537, 1984.

[4] D. C. Tsui, A. C. Gossard, B. F. Field, M. E. Cage, and R. F. Dziuba, "Determination of the fine-structure constant using GaAs- $\mathrm{Al}_{x} \mathrm{Ga}_{1-x} \mathrm{As}$ heterostructures," Phys. Rev. Let., vol. 48, pp. 3-6, 1982.

[5] L. Bliek, E. Braun, H. J. Engelmann, H. Leontiew, F. Melchert, W. Schlapp, B. Stahi, P. Wernecke, and G. Weimann, "Measurements of the quantized Hall resistance $\mathrm{h} / \mathrm{e}^{2}$ with a reproducibility of $10^{-8}$ and its application for a novel determination of the $\mathrm{Ohm}$," PTB-Mitt., 93 1/93, pp. 21-23, 1983.

[6] M. Koyanagi, T. Endo, and A. Nakamura, "Realization of a Josephson potentiometer," in Proc. Precision Measurement and Fundamental Constants II, NBS Spec. Publ. 617, pp. 489-492, 1984.

[7] T. Endo, M. Koyanagi, and A. Nakamura, "High-accuracy Josephson potentiometer," IEEE Trans. Instrum. Meas., vol. IM-32, pp. 267-271, 1983.

[8] J. Clarke, "Advances in SQUID magnetometers," IEEE Trans. Electron Devices, vol. ED-27, pp. 1896-1908, 1980.

\title{
A Sub-PPM Automated 1-10 Volt DC Measuring System
}

\author{
BRUCE F. FIELD, MEMBER, IEEE
}

\begin{abstract}
An automated measuring system has been developed for measuring arbitrary-voltage references in the range of 1-10 V with an inherent measurement accuracy of $\pm 0.22 \mathrm{ppm}(3 \sigma)$. This paper discusses the design and uncertainty analysis of the system and presents data obtained on a Zener voltage reference.
\end{abstract}

\section{INTRODUCTION}

V OLTAGE standards based on solid-state devices are now available with stabilities approaching $1 \mathrm{ppm} /$ year [1]-[4]. These standards typically have their best accuracy at some voltage other than the $1.01-\mathrm{V}$ level of standard cells. In order to separate the performance of the reference device from ensuing buffer amplifiers, it is necessary to measure "peculiar"

Manuscript received August 20, 1984. This paper was supported in part by the Calibration Coordination Group of the U.S. Department of Defense.

The author is with the Electricity Division, National Bureau of Standards, Gaithersburg, MD 20899. voltages, for example, $6.583 \mathrm{~V}$. Typically, Kelvin-Varley dividers are used to measure a wide range of voltages in terms of a standard cell. Accuracies better than one ppm are only achieved by frequent, time consuming, manual calibrations of the divider. As an alternative to the Kelvin-Varley method we have constructed a fully automated, self-calibrating measurement system to measure any arbitrary-voltage within the voltage range of 1-10 V. The total measurement uncertainty in comparing a voltage between 5 and $10 \mathrm{~V}$ to a $1.018 \mathrm{~V}$ standard cell is $\pm 0.22 \mathrm{ppm}$, three standard deviation estimate $(3 \sigma)$.

\section{SYSTEM DESCRIPTION}

The system to be described is essentially a potentiometric technique which allows voltage measurements to be made without loading the source being measured. Since the system was developed to measure Zener reference units and diodes, we will frequently refer to the voltage source being measured as a Zener reference. Fig. 1 is a simplified diagram of the 


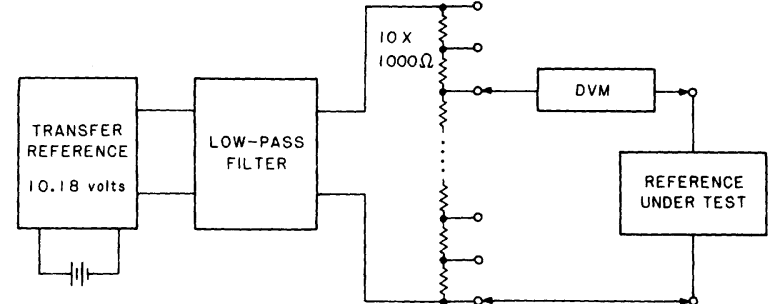

Fig. 1. Block diagram of the measuring system set up to compare a reference-under-test to the calibrated resistor string. Not shown are the crossbar selector switch and desktop computer used to control the switch and DVM.

measuring system. It consists of 10 nominally equal $1000-\Omega$ resistors (resistor string) driven by a $10.18-\mathrm{V}$ battery-operated, solid-state source (transfer reference) with good, short-term stability. The measuring system is used in a two step process. First, the voltage drop across each resistor is determined by comparing it to a standard cell and measuring the microvoltlevel difference voltages with a high-accuracy digital voltmeter (DVM). Then the output voltage developed across $N$ of the resistors is compared to the voltage of the unknown Zener reference using the same DVM. Following this, the voltage drops across the resistors are again compared to the standard cell voltage to estimate the drift in the resistor voltages during the comparison. The voltage of the unknown Zener is obtained from the sum of the voltage drops across the $N$ resistors and the digital voltmeter reading. A low-thermal, crossbar selector switch (not shown in Fig. 1) is used to connect the standard cell and Zener references to the measurement system. Up to 60 voltage sources can be selected for measurement. The DVM and the selector switch are both connected to a desktop computer via the IEEE-488 bus so that the whole measurement process is completely controlled by the computer.

With this system the DVM is used to read only a fraction of the unknown Zener reference voltage which reduces the contribution of the DVM uncertainty to the overall measurement uncertainty. Thus

$$
U=\frac{V_{z}-(N) 1.018}{V_{z}} U_{\mathrm{DVM}}
$$

where $U_{\mathrm{DVM}}$ is the DVM uncertainty expressed as a percentage of reading, $V_{z}$ is the Zener voltage, $N$ is the number of resistors, and $U$ is the final DVM uncertainty as a percentage of the Zener voltage. For a worst case of $V_{z}=5.6 \mathrm{~V}, U=0.09$ $U_{\mathrm{DVM}}$, and for $V_{z}=10 \mathrm{~V}, U=0.02 U_{\mathrm{DVM}}$. Therefore, if the DVM can be calibrated to an accuracy of $1 \mathrm{ppm}$ the worst case error contribution is $0.09 \mathrm{ppm}$. The gain of the DVM is calibrated during the measurements by periodically measuring the $1.018 \mathrm{~V}$ developed across one or more of the resistors. The linearity of the DVM on the $10-\mathrm{V}$ range can be checked by directly measuring the ten voltages, $1.018-10.18 \mathrm{~V}$, developed across the resistors.

\section{TRANSFER REFERENCE}

The transfer reference is a commercial solid-state reference that has been modified to produce $10.18 \mathrm{~V}$ rather than the $10 \mathrm{~V}$ normally supplied. When measurements are being taken, the reference is disconnected from the ac power line and operated from its internal batteries to eliminate coupling to the power line. When the reference is disconnected from the ac power the output voltage is observed to drift down approximately $0.2 \mathrm{ppm}$ during the first hour, apparently caused by temperature changes in the reference. This particular transfer reference has the power transformer in the same enclosure as the reference device and the latter is not temperature controlled. When power is removed from the transformer the internal temperature typically drifts down by about $1^{\circ} \mathrm{C}$ during the first hour. After a one hour "warm up" time the typical output voltage drift is $-0.018 \mathrm{ppm} / \mathrm{h}$. Since the voltage drops across the resistors (and hence the transfer reference), they are calibrated by standard cells before and after Zener measurements, only short-term stability is required of the transfer reference.

\section{Low-Pass Filter}

The transfer reference was found to be sensitive to ac noise coupled into it from the output terminals. If any solid-state voltage reference is not properly isolated from external noise sources, nonlinear circuit elements, including the Zener diode, rectify the ac signals and produce a dc shift in the output voltage [5]. Connecting the DVM directly to the transfer reference produced a dc shift in the output voltage of 0.2 ppm. This was determined by monitoring the reference output voltage with a passive measuring system while connecting the DVM. The value of the dc shift was dependent on the position of the DVM in the measuring circuit, and was extremely reproducible from day-to-day. This produced a systematic error in the calibration of the voltage drops of the 10 resistors. A three-stage $R C$ PI low-pass filter with a cutoff frequency of $400 \mathrm{~Hz}$ was added between the output of the transfer reference and the resistor string to reduce the ac noise. The filter forms part of a voltage divider (with the resistor string) so wire-wound resistors were used for stability and to reduce thermal EMF's. Installation of the filter reduced the worst case dc shift to $0.039 \mathrm{ppm}$. However, since similar shifts occur during determination of the resistor voltages and during the unknown Zener measurements, the dc shift error is partially canceled. When necessary, filters are also added to the unknown Zener references under test.

\section{RESISTOR STRING}

The resistor string driven by the transfer reference consists of 10 nominally equal, stable, $1000-\Omega$ wire-wound resistors. We would have preferred to use lower-valued resistors to reduce measurement errors from leakage currents, but the transfer reference is limited to a load current of slightly more than $1 \mathrm{~mA}$. The resistors are mounted in a temperature-controlled air enclosure, with a simple solid-state sensor and dc amplifier controller to maintain the short-term temperature variations to within $\pm 0.01^{\circ} \mathrm{C}$.

\section{Crossbar Selector Switch}

The crossbar selector switch, a modified commercial product originally intended for telephone applications, permits up to 
60 voltage sources to be compared to the voltage drops across the resistors or intercompared with each other and is a variation of that described in [6]. The switch consists of a matrix of switch contacts that are actuated by magnetically latched solenoids to reduce the power dissipated in the switch. Gold contacts on beryllium copper are used exclusively, and the switch is mounted in a temperature-lagged enclosure with all leads to the switch thermally connected to a large aluminum plate. The crossbar switch is interfaced to the desktop computer via the IEEE bus. A universal coupler converts the 488 bus commands to parallel outputs which are boosted by transistor-drivers to actuate the solenoids with the proper timing sequences. Switch reliability has been excellent, no switch problems having been encountered in two years of operation. Thermal EMF's are periodically checked by shorting the input leads and measuring the voltage with the DVM. Unresolved thermal EMF's are less than $0.030 \mu \mathrm{V}$.

\section{Digital Voltmeter}

A $6 \frac{1}{2}$ digit DVM with $0.1-\mu \mathrm{V}$ resolution on the $0.1-\mathrm{V}$ range is used as the system voltmeter. The voltmeter is programmed by the desktop computer via the IEEE- 488 bus. All voltages are measured by the DVM in both the forward and reverse direction to cancel offset voltages in the DVM. The polarity reversal is done by the crossbar switch. The $0.1-\mathrm{V}$ range is used when calibrating the resistor string voltages against standard cells, and an integration time of $9 \mathrm{~s}$ for each polarity yields a random measurement uncertainty of $0.034 \mu \mathrm{V}(1 \sigma)$. For measurements of Zeners (as shown in Fig. 1) the voltmeter is calibrated and used only on the one volt range.

\section{DESK TOP COMPUTER}

The desktop computer used with the system is programmable only in the Basic language, has 64 kbytes of main memory, and has two 10 Mbyte disks. The large on-line storage capability permits us to maintain a database on measurements made on all the references, examine data, plot control charts, and produce reports as necessary. The system is fully automated with the computer controlling all aspects of the measurement process including operating the crossbar switch, reading the DVM, supplying ac power to the references under test, and reading the temperature of the standard cells.

A number of programs have been developed to automate completely the measurement process. These programs can be used individually or under the control of an interpretative program which calls the programs as overlays (because of limited main memory) and stores the measurement data on a disk. The major tasks include determining the voltage drops across the resistors in terms of a group of standard cells, comparing the voltages across the resistors to a number of unknown Zener references, and producing final calibration reports and control charts on all the Zener references and standard cells.

The interpreter program is very general, permitting any sequence and/or number of measurements to be made based on a set of measurement commands previously stored on the disk. If no changes are to be made to the previous days measurement sequence, performing a complete Zener reference calibration consists of running the program at the start of each day. However, daily changes in the measurement sequence can easily be made, if desired.

The standard cell voltages are compared to the voltages across the resistors using a redundant measurement design. The individual cell and resistor voltages are then determined using a least squares solution to the data. Zener references are identified in the system by a unique serial number with a translation table stored on the disk to equate the serial number to the proper selector switch circuit. Multiple comparisons are made between the Zener reference voltages and the voltages across the resistors to permit estimation of the random measurement uncertainty.

The EMF values assigned the standard cells in terms of the NBS volt are maintained throughout all data files. If later calibrations indicate that small corrections to the cell voltages are necessary, they can be applied to the final Zener voltage results without recalculating all the intermediate data. The latest corrections are maintained on the disk and are applied automatically by the calibration report generator.

\section{Calibration and Sources of Uncertainty}

By far the most critical component of the system is the digital voltmeter. However, in this system the DVM offset and gain are calibrated during the measurements. Provision is also made to calibrate automatically the linearity at 10 points on the $10 . \mathrm{V}$ range by measuring the 10 voltages $1.018,2.036$, $\cdots, 10.18 \mathrm{~V}$ although periodic linearity measurements at 100 points using a Kelvin-Varley divider have shown that it is not necessary to implement this feature. The system was designed principally to measure Zener diode references in the range $5-10 \mathrm{~V}$. Table I lists the sources of uncertainty in the measuring system extrapolated to the worst case unknown voltage $(5.5 \mathrm{~V})$ in the $5-10 \mathrm{~V}$ range. The system may be used over the range $1-5 \mathrm{~V}$ but with somewhat reduced accuracy when the unknown voltage is not near a cardinal value $(1,2,3, \cdots, \mathrm{V})$. The uncertainties in Table I are discussed below.

The DVM gain is measured during the course of the Zener measurements (usually four times) by reading the voltages across two or more individual resistors in the resistor string. From these measurements the gain error of the $1 \mathrm{~V}$ range can be estimated. Two resistors are used for redundancy and to estimate the errors in measuring voltages above ground.

The DVM linearity is periodically checked on the $10-\mathrm{V}$ range using a calibrated, manual, 7-dial Kelvin-Varley divider. A computer-aided system is used to log the data and typically 100 voltages are measured. Measurements performed when the system was first set up indicated no substantial difference in the linearity of the $10-$ and $1-\mathrm{V}$ ranges. The linearity error on the particular DVM being used was typically $0.7 \mathrm{ppm}$ at half-scale input. Measurements on three other meters of the same model have confirmed that the linearity error is typically less than $1 \mathrm{ppm}$ of full scale.

The DVM leakage and bias currents and the standard cell leakage currents were measured directly with an electrometer and their effects on the measurement calculated. The ac effects on the transfer reference were estimated by monitoring changes in its voltage using a passive measurement circuit referenced to a standard cell while the DVM was measuring 
TABLE I

UnCERTAinties IN the AUtomated Measuring System

\begin{tabular}{lc}
\hline Source of uncertainty & $\begin{array}{c}\text { std. dev. estimate } \\
\text { (ppm) }\end{array}$ \\
DVM gain uncertainty & 0.020 \\
DVM linearity uncertainty & 0.062 \\
DVM leakage/bias currents & 0.023 \\
Standard cell leakage currents & 0.006 \\
AC effects on transfer reference & 0.023 \\
Selector switch thermal emfs & 0.013 \\
Random uncertainty in calibrating & 0.007 \\
transfer reference & 0.013 \\
Random uncertainty in calibrating & \\
unknown reference (within day) & 0.075 \\
$\quad$ RSS subtotal & 0.11 \\
RSS total & 0.133 \\
\hline
\end{tabular}

the resistor string voltages. The selector switch thermal EMF's are periodically measured with the DVM by shorting the input leads and measuring the residual voltages. The random uncertainties in calibrating the transfer reference and the unknown references are estimated from the pooled standard deviations of the measurement designs.

Solid-state voltage references sent to NBS for calibration are now calibrated using this system. In this case an additional uncertainty must be included for the uncertainty of the EMF's of the standard cells used to calibrate the system. The 0.11 ppm uncertainty listed in the table is not indicative of the best obtainable accuracy but is rather an estimate of the uncertainty obtained when the standard cells are calibrated in terms of the NBS volt only three times a year. We have recently installed a cable which will allow more frequent calibration of our cells but it is too early to estimate the improvement in the uncertainty using this method.

As far as is known, all systematic errors listed in Table I are independent of each other. Therefore, they are combined in root-sum-square (RSS) fashion along with the random errors.

\section{Results ANd Conclusions}

Measurements of the output of a typical Zener reference are shown in Fig. 2. The reference exhibits a $1 \mathrm{ppm} /$ year drift with a standard deviation of a single observation of $0.07 \mathrm{ppm}$. We have observed that most references measured by the system showed an unexpectedly high correlation in their voltage fluctuations from day-to-day. This same correlation appeared between references near a cardinal value (i.e., $1.018 \mathrm{~V}$ ) where the DVM reading is small, and $10 \mathrm{~V}$ where the DVM reading is

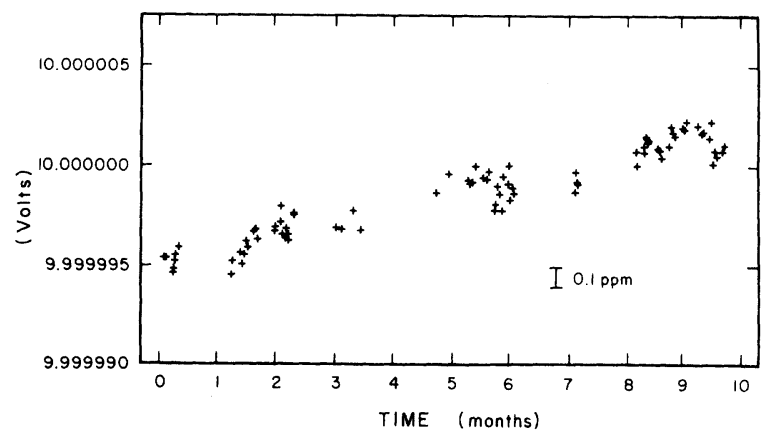

Fig. 2. The measured voltage versus time of a commercial Zener reference standard.

much larger. We concluded that the problem was related to measuring the temperature of our standard cells. Improvements were made to read the temperature automatically at the time of the measurement rather than 1 or $2 \mathrm{~h}$ before, and the correlation was rec uced.

We have developed a fully automated measuring system capable of calibrating an arbitrary-voltage reference with an inherent accuracy of $\pm 0.22 \mathrm{ppm}(3 \sigma)$. It is based on the principle that a properly calibrated digital voltmeter can be used as a transfer device to an accuracy of at least $1 \mathrm{ppm}$. Using this system we have shown that some solid-state voltage references presently available have drift rates as low as $1 \mathrm{ppm} /$ year, nearly as good as standard cells. We have also found that some Zener references shift in voltage by as much as $2 \mathrm{ppm}$ when connected to a DVM and ac filtering must be used to reduce this offset.

In addition, using the principles developed here, possibly in conjunction with fixed ratio voltage dividers, the voltage range can be extended up to $100-1000 \mathrm{~V}$ or down to $100 \mathrm{mV}$, albeit with reduced accuracy. It is also reasonable to use this system as part of a self-calibrating dc reference in an automatic test equipment (ATE) system, or as a high-accuracy automated DVM calibrator.

\section{ACKNOWLEDGMENT}

The author wishes to thank W. G. Eicke for his critical reading of the manuscript and many helpful suggestions.

\section{REFERENCES}

[1] W. Murray and P. Dencher, "High-accuracy voltage transfer using a modified commercial solid-state reference," J. Phys. E.: Sci. Instrum., vol. 17, no. 5, pp. 354-356, 1984.

[2] P. J. Spreadbury and T. E. Everhart, "Ultra-stable portable voltage sources," in Conf. on Electron. Test and Measuring Instrum., IEE Conf. Publ. 174, 1979, pp. 117-120.

[3] K. J. Koep, "The use of a solid state dc voltage transport standard to transfer the dc volt in a regional standard cell MAP program," in Proc. 1984 Meas. Science Conf., 1984, pp.142-146.

[4] L. Huntley, "The Fluke direct voltage maintenance program," in Proc. 1984 Meas. Science Conf., 1984, pp. 147-151.

[5] W. G. Eicke, "The operating characteristics of Zener reference diodes and their measurement," ISA Trans., vol. 3, no. 2, pp. 93-99, Apr. 1964.

[6] D. W. Braudaway and R. E. Kleimann, "A high-resolution prototype system for automatic measurement of standard cell voltages," IEEE Trans. Instrum. Meas., vol. IM-23, pp. 282-286, 1974. 